\title{
Three-loop off-forward evolution kernel for axial-vector operators in Larin's scheme
}

\author{
Vladimir M. Braun, ${ }^{1, *}$ Alexander N. Manashov $\oplus^{2,3, \dagger}$ Sven-O. Moch, ${ }^{2, \$}$ and Matthias Strohmaier ${ }^{1, \S}$ \\ ${ }^{1}$ Institut für Theoretische Physik, Universität Regensburg, D-93040 Regensburg, Germany \\ ${ }^{2}$ II. Institut für Theoretische Physik, Universität Hamburg, D-22761 Hamburg, Germany \\ ${ }^{3}$ St. Petersburg Department of Steklov Mathematical Institute, 191023 St. Petersburg, Russia
}

(Received 7 January 2021; accepted 22 April 2021; published 17 May 2021)

\begin{abstract}
Evolution equations for leading-twist operators in high orders of perturbation theory can be restored from the spectrum of anomalous dimensions and the calculation of the special conformal anomaly at one order less using conformal symmetry of QCD at the Wilson-Fisher critical point at noninteger $d=4-2 \varepsilon$ space-time dimensions. In this work, we generalize this technique to axial-vector operators. We calculate the corresponding three-loop evolution kernels in Larin's scheme and derive explicit expressions for the finite renormalization kernel that describes the difference to the vector case to restore the conventional modified minimal subtraction scheme. The results are directly applicable to deeply virtual Compton scattering and the transition form factor $\gamma^{*} \gamma \rightarrow \pi$.
\end{abstract}

DOI: $10.1103 /$ PhysRevD.103.094018

\section{INTRODUCTION}

The QCD description of hard exclusive reactions in the framework of collinear factorization involves matrix elements of leading-twist operators between hadron states with different momenta-generalized parton distributions (GPDs) or light-cone distribution amplitudes (LCDAs). Such processes are attracting increasing attention because they provide complementary information on the hadron structure as compared to inclusive reactions, and because of the very high quality of experimental data from the JLab $12 \mathrm{GeV}$ upgrade [1], SuperKEKB [2], and, in the future, from the electron ion collider (EIC) [3]. The main motivation for this study is provided by the applications to deeply virtual Compton scattering (DVCS), but the results are also relevant for reactions of the type $\gamma \gamma^{*} \rightarrow \pi$ etc.

The theoretical description of such reactions has to match the experimental accuracy. In particular, evolution equations for GPDs and related quantities have to be derived to similar precision as for the usual parton distributions, currently completely known at next-to-next-leading order (three-loop) $[4,5]$. The difference in these two cases is that for GPDs

\footnotetext{
*vladimir.braun@ur.de

alexander.manashov@desy.de

¥sven-olaf.moch@desy.de

${ }^{\S}$ strohmaiermatthias@gmail.com
}

Published by the American Physical Society under the terms of the Creative Commons Attribution 4.0 International license. Further distribution of this work must maintain attribution to the author(s) and the published article's title, journal citation, and DOI. Funded by SCOAP ${ }^{3}$. (and LCDAs) mixing with operators containing total derivatives must be taken into account. The complete next-toleading order (NLO) (two-loop) evolution kernels for GPDs were calculated long ago [6-8] using an approach developed by Müller [9]. These results were later rederived and confirmed $[10,11]$ by a somewhat different technique $[10,12]$ that makes use of (exact) conformal symmetry of QCD at the Wilson-Fisher critical point in noninteger $d=4-2 \varepsilon$ dimensions. Since renormalization constants in dimensional regularization with minimal subtraction, by construction, do not depend on the space-time dimension, evolution equations in physical QCD at $d=4$ are the same as in the critical theory at $d=4-2 \varepsilon$ and possess a "hidden" conformal symmetry: the evolution kernels in QCD commute with the generators of conformal transformations. These generators in the interacting theory are modified ("deformed") by quantum corrections and the corresponding modification can be calculated order by order in perturbation theory using conformal Ward identities $[8,10,12,13]$. With this approach, the three-loop evolution kernels for GPDs [14] and the corresponding two-loop coefficient functions [15] for DVCS were calculated for flavor-nonsinglet vectorlike distributions.

The extension of this technique to axial-vector distributions requires special considerations due to known issues with the definition of the $\gamma_{5}$-matrix in noninteger dimensions. Having in mind applications to two-photon reactions the well-known prescription by Larin [16] suggests itself; see also Refs. [17-21]. In this work, we study axial-vector operators defined in Larin's scheme under conformal transformations at NLO and, following the method developed in [14], use this result to restore the corresponding 
three-loop evolution kernel. In QCD, it is natural to fix the conventional modified minimal subtraction renormalization scheme $\overline{\mathrm{MS}}$ such that the evolution equations for vector and axial-vector flavor-nonsinglet operators coincide identically. Starting from Larin's prescription, this requires a finite renormalization which is then used to redefine the coefficient functions. This finite renormalization kernel is known for the forward case to three loops [22]; see also [23]. In this paper, we derive the corresponding expression for the general off-forward case.

The presentation is organized as follows. In Sec. II, we introduce axial-vector light-ray operators of leading twist in noninteger dimensions. Section III contains a brief discussion and comparison of the symmetries and renormalization properties of vector and axial-vector operators. We present our results for the two-loop conformal anomaly for axial-vector operators in Sec. IV and for the three-loop evolution kernel in Sec. V. The rotation matrix from Larin's scheme to the $\overline{\mathrm{MS}}$ scheme is given in Sec. VI. We conclude in Sec. VII and the rotation matrix in the local operator basis is presented in the Appendix.

\section{DEFINITION}

GPDs (and LCDAs) are defined as off-forward matrix elements of leading-twist light-ray operators. For the vector case

$$
\mathcal{O}_{V}\left(z_{1}, z_{2}\right)=\bar{q}\left(z_{1} n\right) \gamma_{+}\left[z_{1} n, z_{2} n\right] q\left(z_{2} n\right),
$$

where $n^{\mu}$ is an auxiliary lightlike vector, $n^{2}=0, z_{1,2}$ are real numbers, $\gamma_{+}=\not h=n^{\mu} \gamma_{\mu}$, and $\left[z_{1} n, z_{2} n\right]$ is the Wilson line. The light-ray operator (1) can be viewed as the generating function for local operators, $\mathcal{O}_{V}^{\mu_{1} \ldots \mu_{N}}$ that are symmetric and traceless in all indices $\mu_{1} \ldots \mu_{N}$.

The corresponding axial-vector light-ray operator in four dimensions is naturally given by

$$
\mathcal{O}_{5}\left(z_{1}, z_{2}\right)=\bar{q}\left(z_{1} n\right) \gamma_{+} \gamma_{5}\left[z_{1} n, z_{2} n\right] q\left(z_{2} n\right) .
$$

However, this definition is not suitable for theories in noninteger $d=4-2 \varepsilon$ dimensions as the $\gamma_{5}$-matrix is not defined.

Note that in applications to two-photon reactions one needs the operator product expansion (OPE) of two vector (electromagnetic) currents, which is perfectly well defined in noninteger dimensions. The products of $\gamma$-matrices which occur in loop diagrams can be reduced to the basis of antisymmetric products,

$$
\Gamma_{\mu_{1} \ldots \mu_{n}}=\gamma_{\left[\mu_{1} \ldots \gamma_{\mu_{n}}\right]}
$$

where $[. .$.$] stands for antisymmetrization in the enclosed$ Lorentz indices. It is easy to see that for leading-twist operators the antisymmetric products of more than three $\gamma$-matrices cannot appear (in other words, there are no evanescent operators) so that only one light-ray operator can contribute in addition to (1),

$$
\mathcal{O}_{\mu \nu}\left(z_{1}, z_{2}\right)=\bar{q}\left(z_{1} n\right) \Gamma_{\mu \nu \alpha} n^{\alpha}\left[z_{1} n, z_{2} n\right] q\left(z_{2} n\right) .
$$

In four dimensions, this operator can be rewritten in terms of the axial-vector operator (2),

$\mathcal{O}_{\mu \nu}\left(z_{1}, z_{2}\right)=i \varepsilon_{\mu \nu \alpha \beta} n^{\alpha} \frac{\partial}{\partial n_{\beta}} \int_{0}^{1} d u \mathcal{O}_{5}\left(z_{1} u, z_{2} u\right)+\ldots$,

where ellipses stand for higher-twist terms.

The addenda appear because the operator (4) does not have a definite twist yet. The reason is that when going over to local operators,

$$
n^{\alpha} n^{\nu_{1}} \ldots n^{\nu_{N-1}} \bar{q}(0) \Gamma_{\mu \nu \alpha} D_{\nu_{1}} \ldots D_{\nu_{N-1}} q(0),
$$

the multiplication with the auxiliary lightlike vector $n^{\mu}$ does not yield a traceless result in pairs of indices $\left(\mu, \nu_{k}\right)$ and $\left(\nu, \nu_{k}\right)$. An additional subtraction is needed to separate the leading-twist part. The corresponding condition in the light-ray operator formalism is that the leading-twist-two part of the operator $\mathcal{O}_{\mu \nu}\left(z_{1}, z_{2}\right)$ must obey the constraint

$$
\left.\partial^{\mu} \mathcal{O}_{\mu \nu}\left(z_{1}, z_{2}\right)\right|_{l . t .}=0
$$

For a generic matrix element between states with different momenta, this constraint reduces to

$$
\Delta^{\mu}\left\langle P^{\prime}\left|\mathcal{O}_{\mu \nu}\left(z_{1}, z_{2}\right)\right| P\right\rangle=0, \quad \Delta^{\mu}=\left(P^{\prime}-P\right)^{\mu} .
$$

Since in addition (by construction)

$$
n^{\mu}\left\langle P^{\prime}\left|\mathcal{O}_{\mu \nu}\left(z_{1}, z_{2}\right)\right| P\right\rangle=0,
$$

it follows that the leading-twist part of the operator $\mathcal{O}_{\mu \nu}\left(z_{1}, z_{2}\right)$ corresponds to the transverse components with respect to the $n, \Delta$ plane. Let

$$
\Delta^{\mu}=\alpha n^{\mu}+\beta \bar{n}^{\mu},
$$

with $\bar{n}^{2}=n^{2}=0,(n \bar{n})=1$, and choose two orthogonal unit vectors in transverse directions, $a^{\mu}$ and $b^{\mu}$, such that $(a \cdot n)=(a \cdot \bar{n})=0$ and $(b \cdot n)=(b \cdot \bar{n})=0$.

The leading-twist-two axial-vector operator in noninteger dimensions can be defined as

$\mathcal{O}_{A}\left(z_{1}, z_{2}\right)=a^{\mu} b^{\nu} \mathcal{O}_{\mu \nu}\left(z_{1}, z_{2}\right)=\bar{q}\left(z_{1} n\right) \Gamma_{+}\left[z_{1} n, z_{2} n\right] q\left(z_{2}\right)$,

where 


$$
\Gamma_{+}=a^{\mu} b^{\nu} \Gamma_{\mu \nu \alpha} n^{\alpha}
$$

Note that in four dimensions $a^{\mu} b^{\nu} \varepsilon_{\mu \nu \alpha \sigma} n^{\alpha} \sim n_{\sigma}$ so that $\Gamma_{+} \sim i \gamma_{+} \gamma_{5}$ and the operator in (11) reduces to the one in (2), as desired. Our definition is, of course, a version of Larin's scheme [16].

\section{RENORMALIZATION AND SYMMETRIES}

Here and in what follows we consider QCD in $d=4-2 \varepsilon$ dimensions and tacitly imply that all operators are renormalized in the $\overline{\mathrm{MS}}$ scheme.

Renormalized light-ray operators satisfy the renormalization group $(\mathrm{RG})$ equations,

$$
\left(\mu \frac{\partial}{\partial \mu}+\beta(a) \frac{\partial}{\partial a}+\mathbb{M}_{\mathbf{p}}(a)\right) \mathcal{O}_{\mathbf{p}}\left(z_{1}, z_{2}\right)=0,
$$

where $\mathbf{p}=V, A$, the strong coupling is $a=\alpha_{s} /(4 \pi)$, and $\beta(a)$ is the $d$-dimensional beta function,

$$
\beta(a)=-2 a\left(\varepsilon+\beta_{0} a+\beta_{1} a^{2}+O\left(a^{3}\right)\right),
$$

$\beta_{0}=11 / 3 N_{c}-2 / 3 n_{f}$, etc. for an $\mathrm{SU}\left(N_{c}\right)$ gauge theory with $n_{f}$ quark flavors. At the critical point, the $d$ dimensional beta function vanishes. This can be achieved either by fine-tuning the coupling $a \mapsto a_{*}$ for fixed $\varepsilon=(4-d) / 2$, or fine-tuning $\varepsilon \mapsto \varepsilon_{*}=-\beta_{0} a-\beta_{1} a^{2}-$ $\cdots$ for a fixed value of the coupling. In what follows, we use both notations intermittently.

The evolution kernels $\mathbb{W}_{\mathbf{p}}(a)$ are integral operators in $z_{1}, z_{2}$,

$$
\mathbb{H}_{\mathbf{p}}(a)=\sum_{\ell=1}^{\infty} a^{\ell} \mathfrak{n}_{\mathbf{p}}^{(\ell)}
$$

They can be written in the form

$$
\left[\mathbb{q}_{\mathbf{p}}^{(\ell)} f\right](z)=\int_{0}^{1} d \alpha d \beta h_{\mathbf{p}}^{\ell}(\alpha, \beta) f\left(z_{12}^{\alpha}, z_{21}^{\beta}\right),
$$

where $z$ abbreviates the set of $z_{1,2}$, i.e., $z \equiv\left\{z_{1}, z_{2}\right\}$ and

$$
z_{12}^{\alpha} \equiv z_{1} \bar{\alpha}+z_{2} \alpha, \quad \bar{\alpha} \equiv 1-\alpha
$$

The one-loop kernels for the vector and axial-vector operators coincide, $\mathbb{G}_{V}^{(1)}=\mathbb{G}_{A}^{(1)}$, and are given by the following expression [24]:

$$
\begin{aligned}
{\left[\mathbb{H}_{\mathbf{p}}^{(1)} f\right](z)=} & 4 C_{F}\left\{\frac{1}{2} f(z)-\int_{0}^{1} d \alpha \int_{0}^{\bar{\alpha}} d \beta f\left(z_{12}^{\alpha}, z_{21}^{\beta}\right)\right. \\
& \left.+\int_{0}^{1} d \alpha \frac{\bar{\alpha}}{\alpha}\left(2 f(z)-f\left(z_{12}^{\alpha}, z_{2}\right)-f\left(z_{1}, z_{21}^{\alpha}\right)\right)\right\} .
\end{aligned}
$$

At the classical level, vector (1) and axial-vector light-ray operators (11) transform under conformal transformations in the same way. As a consequence, the one-loop evolution kernels in both cases commute with the canonical generators of the collinear conformal subgroup,

$$
\left[S_{ \pm, 0}, \mathbb{M}_{\mathbf{p}}^{(1)}\right]=0,
$$

where

$$
\begin{aligned}
S_{-} & =-\partial_{z_{1}}-\partial_{z_{2}}, \\
S_{0} & =z_{1} \partial_{z_{1}}+z_{2} \partial_{z_{2}}+2, \\
S_{+} & =z_{1}^{2} \partial_{z_{1}}+z_{2}^{2} \partial_{z_{2}}+2 z_{1}+2 z_{2} .
\end{aligned}
$$

This property follows from the conformal invariance of the QCD Lagrangian at the classical level. Beyond tree level the scale and conformal symmetries are broken by quantum corrections. In noninteger dimensions, however, there exists a nontrivial fixed point, $a=a_{*}$, such that $\beta\left(a_{*}\right)=0$, so that for this special choice of the coupling both scale and conformal invariance of the theory are restored. The symmetry generators in the critical theory, $S_{ \pm, 0}$, satisfy the usual $\mathrm{SL}(2)$ algebra but differ from the canonical generators (20) by quantum corrections. These deformed symmetry generators commute with the evolution kernels,

$$
\left[\mathbf{S}_{\alpha}^{\mathbf{p}}\left(a_{*}\right), \mathbb{H}_{\mathbf{p}}\left(a_{*}\right)\right]=0 .
$$

It can be shown, see Ref. [13] for details, that this modification affects the generators of dilatations and special conformal transformations which take the form

$$
\begin{aligned}
& S_{0}^{\mathbf{p}}=S_{0}+\left(-\varepsilon+\frac{1}{2} \mathbb{M}_{\mathbf{p}}\left(a_{*}\right)\right), \\
& \mathbf{S}_{+}^{\mathbf{p}}=S_{+}+\left(z_{1}+z_{2}\right)\left(-\varepsilon+\frac{1}{2} \mathbb{M}_{\mathbf{p}}\left(a_{*}\right)\right)+z_{12} \Delta_{\mathbf{p}}\left(a_{*}\right),
\end{aligned}
$$

whereas the generator $S_{-}$(corresponding to translations along the light ray) does not receive any corrections, $\mathrm{S}_{-}^{\mathrm{p}}=S_{-}$. These expressions are valid for both cases, $\mathbf{p}=V, A$. Note that the modification of the generator of dilatations is expressed in terms of the evolution kernel whereas for the conformal transformations there is an additional contribution. This additional term is usually 
referred to as the conformal anomaly. It can be calculated order by order in perturbation theory,

$$
\Delta_{\mathbf{p}}\left(a_{*}\right)=\sum_{\ell} a_{*}^{\ell} \Delta_{\mathbf{p}}^{(\ell)},
$$

from the conformal Ward identities for the corresponding light-ray operators; see $[8,13]$ for details. At one-loop order, the conformal anomaly for the vector and axialvector operators coincide, $\Delta_{V}^{(1)}=\Delta_{A}^{(1)}$. The corresponding expression was first obtained in Ref. [8],

$\left[\Delta_{\mathbf{p}}^{(1)} f\right](z)=\int_{0}^{1} d \alpha \omega^{(1)}(\alpha)\left[f\left(z_{12}^{\alpha}, z_{2}\right)-f\left(z_{1}, z_{21}^{\alpha}\right)\right]$,

where the weight function $\omega^{(1)}$ reads

$$
\omega^{(1)}(\alpha)=-2 C_{F}\left(\frac{\bar{\alpha}}{\alpha}+\ln \alpha\right) .
$$

The two-loop conformal anomaly for the vector case, $\Delta_{V}^{(2)}$, was derived in [13], the resulting expression being too lengthy to be presented here.

It is convenient to write the evolution kernels, $\mathbb{U}_{\mathbf{p}}$, as a sum of two terms of which the first one is invariant and the second one is noninvariant with respect to the canonical symmetry transformations. Suppressing the $V / A$ subscript, we define

$$
\mathbb{H}=\mathbb{\boxplus}^{\text {inv }}+\mathbb{\boxplus}^{\text {noninv }}, \quad\left[S_{\alpha}, \mathbb{W}^{\text {inv }}\right]=0 .
$$

It was shown in [8-10] that the operator $\mathbb{Q}^{\text {noninv }}$ at $\ell$-loop order is completely determined (up to invariant terms) by the conformal anomaly at one order less, $\ell-1$. Once this noninvariant operator is fixed, the invariant part $\mathbb{U}^{\text {inv }}$ can be restored from the corresponding anomalous dimensions (at $\ell$ loops). These are known for vector and axial-vector operators (in Larin's scheme) to three-loop accuracy [4,22].

Since $\mathbb{U}_{V}^{(1)}=\mathbb{T}_{A}^{(1)}$ and $\Delta_{V}^{(1)}=\Delta_{A}^{(1)}$, the generators of special conformal transformations for vector and axialvector operators coincide to this accuracy. From the commutation relations (19), it follows then that the leading two-loop term for the difference of the evolution kernels,

$$
\mathbb{\boxplus}_{A-V}(a)=\mathbb{\boxplus}_{A}(a)-\mathbb{\boxplus}_{V}(a)=a^{2} \mathbb{\boxplus}_{A-V}^{(2)}+O\left(a^{3}\right),
$$

is a canonically invariant operator. It is completely determined by its spectrum which is given by the difference of anomalous dimensions of vector and axial-vector operators, $\gamma_{V}(N)-\gamma_{A}(N)$, where $N$ denotes the spin of the operator. We obtain after a short calculation

$$
\left[\mathbb{W}_{A-V}^{(2)} f\right](z)=16 C_{F} \beta_{0} \int_{0}^{1} d \alpha \int_{0}^{\bar{\alpha}} d \beta f\left(z_{12}^{\alpha}, z_{21}^{\beta}\right) .
$$

In order to calculate the difference of the vector an axialvector kernels to $O\left(a^{3}\right)$, one needs the difference in the corresponding conformal anomalies at two loops.

\section{CONFORMAL ANOMALY FOR AXIAL-VECTOR OPERATORS TO $O\left(a^{2}\right)$}

The calculation of the conformal anomaly for vector operators is discussed at length in $[10,13]$. The only modification for the axial-vector operators is to replace the $\gamma_{+}$matrix by $\Gamma_{+}$defined in Eq. (12) in the operator vertex in the corresponding diagrams. Simplifying the numerators, one uses the following properties of the $\gamma_{+}$ matrix in $d=4-2 \varepsilon$ :

$$
\gamma_{+} \gamma_{+}=0, \quad \gamma^{\mu} \gamma_{+} \gamma_{\mu}=-2(1-\varepsilon) \gamma_{+} .
$$

The corresponding identities for $\Gamma_{+}$take the form

$$
\gamma_{+} \Gamma_{+}=\Gamma_{+} \gamma_{+}=0, \quad \gamma^{\mu} \Gamma_{+} \gamma_{\mu}=2(1+\varepsilon) \Gamma_{+} .
$$

The diagrams to be calculated are shown in Fig. 2, Ref. [13]. One is interested in the residues of the simple poles in $\varepsilon$. It is easy to see that the replacement $\gamma_{+} \rightarrow \Gamma_{+}$ does not affect the diagrams with one interacting quark in Figs. 2(a)-2(g). For the remaining diagrams in Figs. 2(h) -2 (p), the modifications due to the substitution $\gamma_{+} \rightarrow \Gamma_{+}$ can easily be tracked down and are related to the factors $(1-\varepsilon)^{k}$ vs $(1+\varepsilon)^{k}$ arising in the calculation. The expression for a generic diagram has the form

$$
\gamma^{\alpha_{1}} \ldots \gamma^{\alpha_{2 k}} \Gamma \gamma_{\beta_{1}} \ldots \gamma_{\beta_{2 m}}\left(\frac{1}{\varepsilon^{2}}\left(T_{1}\right)_{\alpha_{1} \ldots \alpha_{2 k}}^{\beta_{1} \ldots \beta_{2 m}}+\frac{1}{\varepsilon}\left(T_{2}\right)_{\alpha_{1} \ldots \alpha_{2 k}}^{\beta_{1} \ldots \beta_{2 m}}\right),
$$

where $\Gamma=\gamma_{+}$or $\Gamma_{+}$and $T_{1,2}$ are certain tensors which depend on the external momenta. Since we are interested in the $1 / \varepsilon$ pole only, contracting the Lorentz indices on the string of $\gamma$-matrices with $T_{2}$, one can use the fourdimensional algebra and replace $\Gamma_{+}$by $i \gamma_{+} \gamma_{5}$. The $1 / \varepsilon$ contributions due to $T_{2}$ are therefore the same for the vector and axial-vector operators. Hence, one needs to consider the double-pole contributions of $T_{1}$ only, which are related to divergent subgraphs and are easy to calculate. Such double-pole contributions for a given diagram take the form

$$
\frac{1}{\varepsilon^{2}} \sum_{m} D_{m}(\varepsilon \pm 1)^{2 m}
$$

with coefficients $D_{m}$, where \pm corresponds to the axial and vector cases, respectively, and we take into account that the factors $(\varepsilon \pm 1)$ can only appear in even powers since the residues of the double poles have to coincide. Thus, the difference between the axial-vector and vector operators for a given diagram takes the form 


$$
\frac{1}{\varepsilon} \sum_{m} 4 m D_{m}+O\left(\varepsilon^{0}\right)
$$

The coefficients $D_{m}$ are sufficiently easy to calculate. We have checked that the difference of the two-loop evolution kernels (27) calculated in this way diagrammatically coincides with the result in Eq. (28).

The difference of two-loop conformal anomalies (22) for axial-vector and vector operators, $\Delta_{A-V}^{(2)}=\Delta_{A}^{(2)}-\Delta_{V}^{(2)}$, can be written as a sum of two terms

$$
\Delta_{A-V}^{(2)}=\frac{1}{4}\left[\mathbb{H}_{A-V}^{(2)}, z_{1}+z_{2}\right]+\hat{\Delta}_{A-V}^{(2)}
$$

where the operator $\hat{\Delta}_{A-V}^{(2)}$ is defined as

$$
\left[\hat{\Delta}_{A-V}^{(2)} f\right](z)=\int_{0}^{1} d \alpha \int_{0}^{\bar{\alpha}} d \beta\left[\omega^{(2)}(\alpha)-\omega^{(2)}(\beta)\right] f\left(z_{12}^{\alpha}, z_{21}^{\beta}\right)
$$

with the kernel

$$
\omega^{(2)}(\alpha)=8 C_{F}^{2}\left[\frac{3}{2} \alpha+\ln \alpha\left(\frac{1}{\bar{\alpha}}-\bar{\alpha}\right)+\bar{\alpha} \ln \bar{\alpha}\right] .
$$

Contributions with the color factors $\beta_{0} C_{F}$ and $C_{F} C_{A}$ cancel in the sum of all diagrams.

\section{THREE-LOOP EVOLUTION KERNEL}

The canonically noninvariant part of the evolution kernel is completely determined by the commutation relations in Eq. (21). The analysis of these equations becomes much simpler after making a similarity transformation of the operators at the intermediate step [14],

$$
\mathrm{S}_{\alpha}^{\mathbf{p}}=\mathrm{U}_{\mathbf{p}}^{-1} \mathbf{S}_{\alpha}^{\mathbf{p}} \mathrm{U}_{\mathbf{p}}, \quad \mathbb{H}_{\mathbf{p}}=\mathrm{U}_{\mathbf{p}}^{-1} \mathbf{H}_{\mathbf{p}} \mathrm{U}_{\mathbf{p}}
$$

where the rotation matrix $U_{\mathbf{p}}$ is chosen in such a way that the new (boldfaced) symmetry generators do not include the conformal anomaly term,

$$
\begin{aligned}
\mathbf{S}_{-}^{\mathbf{p}} & =S_{-}, \\
\mathbf{S}_{0}^{\mathbf{p}} & =S_{0}+\left(-\varepsilon+\frac{1}{2} \mathbf{H}_{\mathbf{p}}\right), \\
\mathbf{S}_{+}^{\mathbf{p}} & =S_{+}+\left(z_{1}+z_{2}\right)\left(-\varepsilon+\frac{1}{2} \mathbf{H}_{\mathbf{p}}\right) .
\end{aligned}
$$

The rotation matrix can be written in the form [14]

$$
\mathrm{U}_{\mathbf{p}}=\exp \left\{a \mathbb{X}_{\mathbf{p}}^{(1)}+a^{2} \mathbb{X}_{\mathbf{p}}^{(2)}+O\left(a^{3}\right)\right\}
$$

The one-loop $\mathbb{X}$-kernels for the vector and axial-vector operators are equal to each other,

$$
\begin{aligned}
& {\left[\mathbb{X}_{\mathbf{p}}^{(1)} f\right](\boldsymbol{z})} \\
& \quad=2 C_{F} \int_{0}^{1} d \alpha \frac{\ln \alpha}{\alpha}\left[2 f(\boldsymbol{z})-f\left(z_{12}^{\alpha}, z_{2}\right)-f\left(z_{1}, z_{21}^{\alpha}\right)\right]
\end{aligned}
$$

and obey the equation $\left[S_{+}, \mathbb{X}_{\mathbf{p}}^{(1)}\right]=z_{12} \Delta_{\mathbf{p}}^{(1)}$. The expression for the two-loop kernel for the vector case, $\mathbb{X}_{V}^{(2)}$, can be found in [14]. The difference

$$
\mathbb{X}_{A-V}^{(2)}=\mathbb{X}_{A}^{(2)}-\mathbb{X}_{V}^{(2)}
$$

is defined as a solution of the equation [25]

$$
\left[S_{+}, \mathbb{X}_{A-V}^{(2)}\right]=\frac{1}{4}\left[\mathbb{H}_{A-V}^{(2)}, z_{1}+z_{2}\right]+z_{12} \hat{\Delta}_{A-V}^{(2)}
$$

The solution can be written as a sum of two terms

$$
\mathbb{X}_{A-V}^{(2)}=\frac{1}{4} \mathbb{T}_{A-V}^{(2)}+\Delta \mathbb{X}_{A-V}^{(2)}
$$

corresponding to the two contributions on the rhs of Eq. (41), respectively. We find

$$
\left[\mathbb{T}_{A-V}^{(2)} f\right](z)=16 C_{F} \beta_{0} \int_{0}^{1} d \alpha \int_{0}^{\bar{\alpha}} d \beta \ln (1-\alpha-\beta) f\left(z_{12}^{\alpha}, z_{21}^{\beta}\right)
$$

and

$$
\left[\Delta \mathbb{X}_{A-V}^{(2)} f\right](\boldsymbol{z})=\int_{0}^{1} d \alpha \int_{0}^{\bar{\alpha}} d \beta[\chi(\alpha)+\chi(\beta)] f\left(z_{12}^{\alpha}, z_{21}^{\beta}\right),
$$

with the weight function

$$
\chi(\alpha)=8 C_{F}^{2}\left[-\frac{1}{2} \ln \bar{\alpha}+\frac{\alpha}{\bar{\alpha}} \ln \alpha+\mathrm{Li}_{2}(\bar{\alpha})-\mathrm{Li}_{2}(\alpha)\right]
$$

Using these results for the rotation matrix (38) and the expression given in Ref. [14] [Eq. (3.12)] for the three-loop kernel $\mathfrak{H}_{V}^{(3)}$, we can restore the $O\left(a^{3}\right)$ contribution to the difference $\mathbb{H}_{A-V}$, Eq. (27). The result reads

$$
\begin{aligned}
\mathfrak{H}_{A-V}^{(3)}= & \mathbb{H}_{A-V}^{(3), \text { inv }}+\frac{1}{2} \mathbb{T}_{V}^{(1)} \mathbb{H}_{A-V}^{(2)}+\mathbb{T}_{A-V}^{(2)}\left(\beta_{0}+\frac{1}{2} \mathbb{H}_{V}^{(1)}\right) \\
& +\left[\mathbb{H}_{V}^{(1)}, \mathbb{X}_{A-V}^{(2)}\right]+\left[\mathbb{H}_{A-V}^{(2)}, \mathbb{X}_{V}^{(1)}\right]
\end{aligned}
$$

The expressions for $\mathbb{T}_{A-V}^{(2)}, \mathbb{H}_{A-V}^{(2)}, \mathbb{H}_{V}^{(1)}, \mathbb{X}_{V}^{(1)}, \mathbb{X}_{A-V}^{(2)}$ are given above and 


$$
\begin{aligned}
{\left[\mathbb{T}_{V}^{(1)} f\right](\boldsymbol{z})=} & -4 C_{F}\left\{\int_{0}^{1} d \alpha \frac{\bar{\alpha} \ln \bar{\alpha}}{\alpha}\left[f\left(z_{12}^{\alpha}, z_{2}\right)+f\left(z_{1}, z_{21}^{\alpha}\right)\right]\right. \\
& \left.+\int_{0}^{1} d \alpha \int_{0}^{\bar{\alpha}} d \beta \ln (1-\alpha-\beta) f\left(z_{12}^{\alpha}, z_{21}^{\beta}\right)\right\}
\end{aligned}
$$

is defined as a solution of the equation

$$
\left[S_{+}, \mathbb{T}_{V}^{(1)}\right]=\left[\mathbb{H}_{V}^{(1)}, z_{1}+z_{2}\right] .
$$

The last missing element in Eq. (46) is the invariant kernel $\mathbb{Q} \mathbb{Q}_{A-V}^{(3) \text {,inv }}$. It is completely determined by its eigenvalues

$$
\mathbb{Q}_{A-V}^{(3), \text { inv }}\left(z_{1}-z_{2}\right)^{N-1}=\gamma_{A-V}^{(3) \text {,inv }}(N)\left(z_{1}-z_{2}\right)^{N-1},
$$

which can be found as

$$
\gamma_{A-V}^{(3), \text { inv }}(N)=\gamma_{A-V}^{(3)}(N)-\gamma_{A-V}^{(3), \text { ninv }}(N) .
$$

Here $\gamma_{A-V}^{(3)}(N)$ is the difference of the three-loop anomalous dimensions of vector and axial-vector operators (i.e., the eigenvalues of the kernel $\left.\mathbb{U}_{A-V}^{(3)}\right)$ and $\gamma_{A-V}^{(3), \text { ninv }}(N)$ are the eigenvalues of the noninvariant operators on the rhs of Eq. (46). One easily finds (note that the commutator terms do not contribute to $\left.\gamma_{A-V}^{(3) \text {,ninv }}(N)\right)$

$$
\gamma_{A-V}^{(3), \text { ninv }}(N)=8 \beta_{0} C_{F} \frac{d}{d N}\left(\frac{\gamma_{V}^{(1)}(N)+2 \beta_{0}}{N(N+1)}\right),
$$

where

$$
\gamma_{V}^{(1)}(N)=4 C_{F}\left(S_{1}(N+1)+S_{1}(N-1)-\frac{3}{2}\right)
$$

is the eigenvalue of the one-loop kernel $\mathbb{q}_{V}^{(1)}$ where $S_{\vec{m}}(N)$ are harmonic sums, cf. Ref. [26]. The difference in the three-

loop anomalous dimensions, $\gamma_{A-V}^{(3)}(N)$, was calculated in Ref. [22]. The result reads

$$
\gamma_{A-V}^{(3)}(N)=-2 \int_{0}^{1} d x x^{N-1} P_{A-V}^{(3)}(x),
$$

with the splitting function written in terms of two functions, $z_{\mathrm{ns}}^{(1)}(x), z_{\mathrm{ns}}^{(2)}(x)$, and their convolutions “ $\otimes$,

$P_{A-V}^{(3)}(x)=\beta_{1} z_{\mathrm{ns}}^{(1)}(x)-\beta_{0}\left(\left[z_{\mathrm{ns}}^{(1)} \otimes z_{\mathrm{ns}}^{(1)}\right](x)-2 z_{\mathrm{ns}}^{(2)}(x)\right)$,

see [22] [Eqs. (A.1), (A.2)]. Starting from these expressions, we find the eigenvalues of the invariant kernel after some algebra as

$$
\begin{aligned}
\gamma_{A-V}^{(3), \text { inv }}(N)= & \frac{16 C_{F} \beta_{1}}{N(N+1)}+16 C_{F} \beta_{0}\left\{\frac{5}{3} \beta_{0} \frac{1}{N(N+1)}\right. \\
& +C_{A}\left(\frac{(-1)^{N-1} 2\left(2 S_{-2}(N)+\zeta_{2}\right)}{N(N+1)}-\frac{2 \zeta_{2}}{N(N+1)}\right. \\
& \left.+\frac{16}{3} \frac{1}{N(N+1)}+\frac{6}{N^{2}(N+1)^{2}}+\frac{4}{N^{3}(N+1)^{3}}\right) \\
& -4 C_{F}\left(\frac{(-1)^{N-1}\left(2 S_{-2}(N)+\zeta_{2}\right)}{N(N+1)}+\frac{1}{N(N+1)}\right. \\
& \left.\left.+\frac{13}{4} \frac{1}{N^{2}(N+1)^{2}}+\frac{2}{N^{3}(N+1)^{3}}\right)\right\} .
\end{aligned}
$$

It can easily be verified that this expression possesses the so-called reciprocity property [27-30]: its asymptotic expansion at large $N$ is invariant under the substitution $N \rightarrow-N-1$.

The last step is to restore the invariant operator $\mathbb{W}_{A-V}^{(3) \text {,inv }}$ from its spectrum. Any invariant operator can be written in the form

$$
\left[\mathbb{R}^{\text {inv }} f\right]\left(z_{1}, z_{2}\right)=\int_{0}^{1} d \alpha \int_{0}^{\bar{\alpha}} d \beta h(\tau) f\left(z_{12}^{\alpha}, z_{21}^{\beta}\right),
$$

where $\tau=\alpha \beta / \bar{\alpha} \bar{\beta}$ is the so-called conformal ratio. The eigenvalues are given by moments of the function $h(\tau)$,

$$
\gamma^{\mathrm{inv}}(N)=\int_{0}^{1} d \alpha \int_{0}^{\bar{\alpha}} d \beta h(\tau)(1-\alpha-\beta)^{N-1},
$$

so that $h(\tau)$ can be restored uniquely by a Mellin transform. Since the expressions for $\gamma_{A-V}^{(3) \text {,inv }}(N)$ are relatively simple, this calculation is rather straightforward. We find

$$
\begin{aligned}
h_{A-V}^{(3) \text {,inv }}(\tau)= & 16 C_{F}\left\{\beta_{1}+\beta_{0}\left(\frac{5}{3} \beta_{0}+4 C_{F}\left[\frac{1}{4} \ln \bar{\tau}-\zeta_{2}+\frac{5}{3}\right]\right.\right. \\
& \left.\left.+\frac{2}{N_{c}}\left[\operatorname{Li}_{2}(\tau)-\zeta_{2}+\ln ^{2} \bar{\tau}-\ln \bar{\tau}+\frac{8}{3}\right]\right)\right\} .
\end{aligned}
$$

With this last piece, the difference in the three-loop evolution kernel, $\mathbb{a}_{A-V}^{(3)}$ and, therefore, also in the axialvector kernel $\mathbb{W}_{A}^{(3)}$ itself, is completely fixed.

\section{MATCHING}

Vector and axial-vector operators in the $d$-dimensional theory at the critical point have different scaling dimensions, i.e., different evolution equations in the chosen scheme for $\gamma_{5}$. In four dimensions, this difference is avoidable: 
There exist four-dimensional regularization schemes in which the (flavor-nonsinglet) vector and axial-vector operators satisfy the same evolution equation. Hence, it is possible to define a new operator $\mathcal{O}_{A}(z) \mapsto \mathcal{O}_{5}(z)$ by the transformation (finite renormalization)

$$
\mathcal{O}_{5}(z)=\mathcal{U} \mathcal{O}_{A}(z)
$$

such that $\mathcal{O}_{5}(z)$ obeys the same evolution equation as the vector operator $\mathcal{O}_{V}(\boldsymbol{z})$ in Eq. (1), which is convenient for applications and conventionally defines the $\overline{\mathrm{MS}}$ scheme. In this section, we derive the explicit expression for the matching kernel $\mathcal{U}$.

Inserting Eq. (59) in Eq. (13), it is easy to see that the operator $\mathcal{O}_{5}(z)$ satisfies the RG equation (in $d=4$ )

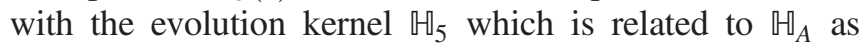
follows:

$\mathcal{U}^{-1}(a) \mathbb{\boxplus}_{5}(a) \mathcal{U}(a)+\mathcal{U}^{-1}(a) \beta(a) \partial_{a} \mathcal{U}(a)=\mathbb{\boxplus}_{A}(a)$.

We require that $\mathbb{U}_{5}(a)=\mathbb{U}_{V}(a)$ and make an ansatz for the $\mathcal{U}$-kernel in the form

$$
\mathcal{U}(a)=\mathrm{P} \exp \left\{\int_{0}^{a} \frac{d s}{\beta(s)} V(s)\right\}
$$

so that $\beta(a) \partial_{a} \mathcal{U}(a)=V(a) \mathcal{U}(a)$ and Eq. (60) takes the form

$$
\mathbb{H}_{V}(a)+V(a)=\mathcal{U}(a) \mathbb{H}_{A}(a) \mathcal{U}^{-1}(a) .
$$

Expanding $V(a)=a^{2} V_{1}+a^{3} V_{2}+O\left(a^{4}\right)$, one obtains

$\mathcal{U}(a)=1-\frac{a}{2 \beta_{0}} V_{1}-\frac{a^{2}}{4 \beta_{0}}\left(V_{2}-\frac{1}{2 \beta_{0}} V_{1}^{2}-\frac{\beta_{1}}{\beta_{0}} V_{1}\right)+O\left(a^{3}\right)$,

and from (62)

$$
\begin{aligned}
V(a)= & a^{2} \mathbb{T}_{A-V}^{(2)}+a^{3} \mathbb{H}_{A-V}^{(3)}+\frac{a^{2}}{2 \beta_{0}}\left[\mathbb{H}_{A}^{(1)}, V_{1}\right] \\
& +\frac{a^{3}}{2 \beta_{0}}\left(\left[\mathbb{H}_{A}^{(2)}, V_{1}\right]+\frac{1}{2}\left[\mathbb{R}_{A}^{(1)}, V_{2}\right]\right)+O\left(a^{4}\right) .
\end{aligned}
$$

Comparing the leading contributions $O\left(a^{2}\right)$ on both sides of this relation, one gets

$$
V_{1}=\mathbb{U}_{A-V}^{(2)}+\frac{1}{2 \beta_{0}}\left[\mathbb{\boxplus}_{A}^{(1)}, V_{1}\right] .
$$

Note that $\mathbb{U}_{A-V}^{(2)} \propto \beta_{0}$, cf. Eq. (28). Assuming that $V_{1}$ cannot contain terms $\sim 1 / \beta_{0}$, the only possibility to match the powers of $\beta_{0}$ in this relation is to require that

$$
V_{1}=\mathbb{H}_{A-V}^{(2)} \quad \text { and } \quad\left[\mathbb{H}_{A}^{(1)}, V_{1}\right]=0 .
$$

Note that these two conditions are self-consistent since $\mathbb{G}_{A-V}^{(2)}$ is an invariant operator; any two invariant operators commute.

Next, comparing the $O\left(a^{3}\right)$ contributions, one obtains an equation for $V_{2}$,

$$
V_{2}+\frac{1}{4 \beta_{0}}\left[V_{2}, \mathbb{M}_{A}^{(1)}\right]=\mathbb{\boxplus}_{A-V}^{(3)}-\frac{1}{2 \beta_{0}}\left[V_{1}, \mathbb{M}_{A}^{(2)}\right] .
$$

The kernel $\mathbb{\boxplus}_{A-V}^{(3)}$ is given in Eq. (46), $\mathbb{\boxplus}_{A}^{(1)}=\mathbb{U}_{V}^{(1)}$; see Eq. (18) and Ref. [14] [Eq. (3.12)],

$\mathbb{M}_{A}^{(2)}=\mathbb{U}_{A}^{(2), \text { inv }}+\mathbb{T}_{1}\left(\beta_{0}+\frac{1}{2} \mathbb{\boxplus}_{V}^{(1)}\right)+\left[\mathbb{\boxplus}_{V}^{(1)}, \mathbb{X}_{V}^{(1)}\right]$,

with $\mathbb{U}_{A}^{(2), \text { inv }}=\mathbb{G}_{V}^{(2), \text { inv }}+\mathbb{G}_{A-V}^{(2)}$.

Since $V_{1}$ and $\mathbb{H}_{V}^{(1)}$ are invariant operators, the invariant parts of $V_{2}$ and $\mathbb{U}_{A}^{(2)}$ drop out from the commutators on the lhs and rhs of Eq. (67), respectively. Thus, if we write

$$
V_{2}=\mathbb{H}_{A-V}^{(3) \text { inv }}+V_{2}^{\prime},
$$

the terms in $\mathbb{q}_{A-V}^{(3) \text {,inv }}$ cancel out and one ends up with the equation for the noninvariant part of the two-loop matching kernel,

$$
\begin{aligned}
& V_{2}^{\prime}+\frac{1}{4 \beta_{0}}\left[V_{2}^{\prime}, \mathbb{\boxplus}_{V}^{(1)}\right]=\frac{1}{2} \mathbb{T}_{V}^{(1)} \mathbb{\boxplus}_{A-V}^{(2)}+\mathbb{T}_{A-V}^{(2)}\left(\beta_{0}+\frac{1}{2} \mathbb{\boxplus}_{V}^{(1)}\right) \\
& +\frac{1}{4}\left[\mathbb{H}_{V}^{(1)}, \mathbb{T}_{A-V}^{(2)}\right] \\
& -\frac{1}{2 \beta_{0}}\left[\mathbb{T}_{A-V}^{(2)}, \mathbb{T}_{V}^{(1)}\right]\left(\beta_{0}+\frac{1}{2} \mathbb{\boxplus}_{V}^{(1)}\right) \\
& +\left[\mathbb{H}_{V}^{(1)}, \Delta \mathbb{X}_{A-V}^{(2)}\right]+\left[\mathbb{H}_{A-V}^{(2)}, \mathbb{X}_{V}^{(1)}\right] \\
& -\frac{1}{2 \beta_{0}}\left[\mathbb{H}_{A-V}^{(2)},\left[\mathbb{H}_{V}^{(1)}, \mathbb{X}_{V}^{(1)}\right]\right] \text {. }
\end{aligned}
$$

The expression on the rhs of this equation is a second order polynomial in $\beta_{0}$ and we expect the matching kernel $\mathcal{U}$ and, hence, $V(a)$ to be polynomials in $\beta_{0}$ as well (e.g., since Feynman diagrams are trivially polynomials in the number of flavors $n_{f}$ ).

We write

$$
V_{2}^{\prime}=\beta_{0}^{2} V_{2,2}^{\prime}+\beta_{0} V_{2,1}^{\prime}+V_{2,0}^{\prime}
$$

and collect the contributions of different powers of $\beta_{0}$ separately. One obtains four equations, 


$$
\begin{aligned}
& \left(\beta_{0}\right)^{2}: \beta_{0}^{2} V_{2,2}^{\prime}=\beta_{0} \mathbb{T}_{A-V}^{(2)}, \\
& \left(\beta_{0}\right)^{1}: \beta_{0} V_{2,1}^{\prime}+\frac{\beta_{0}}{4}\left[V_{2,2}^{\prime}, \mathbb{\boxplus}_{V}^{(1)}\right] \\
& =\frac{1}{2} \mathbb{T}_{V}^{(1)} \mathbb{\boxplus}_{A-V}^{(2)}+\frac{1}{2} \mathbb{T}_{A-V}^{(2)} \llbracket \mathbb{\boxplus}_{V}^{(1)}+\frac{1}{4}\left[\mathbb{G}_{V}^{(1)}, \mathbb{T}_{A-V}^{(2)}\right] \\
& -\frac{1}{2}\left[\mathbb{W}_{A-V}^{(2)}, \mathbb{T}_{V}^{(1)}\right]+\left[\mathbb{W}_{A-V}^{(2)}, \mathbb{X}_{V}^{(1)}\right], \\
& \left(\beta_{0}\right)^{0}: V_{2,0}^{\prime}+\frac{1}{4}\left[V_{2,1}^{\prime}, \mathbb{H}_{V}^{(1)}\right] \\
& =\left[\mathbb{T}_{V}^{(1)}, \Delta \mathbb{X}_{A-V}^{(2)}\right]-\frac{1}{4 \beta_{0}}\left[\mathbb{\boxplus}_{A-V}^{(2)}, \mathbb{T}_{V}^{(1)}\right] \mathbb{\boxplus}_{V}^{(1)} \\
& -\frac{1}{2 \beta_{0}}\left[\mathbb{H}_{A-V}^{(2)},\left[\mathbb{H}_{V}^{(1)}, \mathbb{X}_{V}^{(1)}\right]\right], \\
& \left(\beta_{0}\right)^{-1}: \frac{1}{4 \beta_{0}}\left[V_{2,0}^{\prime}, \mathbb{W}_{V}^{(1)}\right]=0 .
\end{aligned}
$$

$$
\mathbb{F}=\Delta \mathbb{X}_{A-V}^{(2)}-\frac{1}{8 \beta_{0}}\left(2\left[\mathbb{W}_{A-V}^{(2)}, \mathbb{X}_{V}^{(1)}\right]-\mathbb{T}_{V}^{(1)} \mathbb{T}_{A-V}^{(2)}+\mathbb{T}_{A-V}^{(2)} \mathbb{\boxplus}_{V}^{(1)}\right)
$$

The equation $\left[\mathbb{H}_{V}^{(1)}, \mathbb{F}\right]=0$ implies that $\mathbb{F}$ is an invariant operator and, therefore, it commutes with the canonical conformal symmetry generators, $\left[S_{+}, \mathbb{F}\right]=0$. Since $\left[S_{+}, \Delta \mathbb{X}_{A-V}^{(2)}\right]=z_{12} \hat{\Delta}_{A-V}^{(2)}$, cf. Eq. (41), it follows from $\left[S_{+}, \mathbb{F}\right]=0$ that

$$
\begin{aligned}
z_{12} \hat{\Delta}_{A-V}^{(2)}= & \frac{1}{8 \beta_{0}}\left(2\left[\mathbb{\boxplus}_{A-V}^{(2)}, z_{12} \Delta_{+}^{(1)}\right]-\left[\mathbb{\boxplus}_{V}^{(1)}, z_{1}+z_{2}\right] \mathbb{\boxplus}_{A-V}^{(2)}\right. \\
& \left.+\left[\mathbb{G}_{A-V}^{(2)}, z_{1}+z_{2}\right] \mathbb{G}_{V}^{(1)}\right) .
\end{aligned}
$$

From Eqs. (72), (73), and (75), we obtain

$$
\begin{gathered}
\beta_{0} V_{2,2}^{\prime}=\mathbb{T}_{A-V}^{(2)}, \\
\beta_{0} V_{2,1}^{\prime}=\frac{1}{2} \mathbb{T}_{V}^{(1)} \mathbb{T}_{A-V}^{(2)}+\frac{1}{2} \mathbb{T}_{A-V}^{(2)} \mathbb{\boxplus}_{V}^{(1)}+\left[\mathbb{W}_{A-V}^{(2)}, \mathbb{X}_{V}^{(1)}\right], \\
V_{2,0}^{\prime}=0 .
\end{gathered}
$$

The first two expressions follow readily from Eqs. (72) and (73), respectively. For the last one, from Eq. (75), we conclude that $\left[V_{2,0}^{\prime}, \mathbb{M}_{V}^{(1)}\right]=0$, i.e., $V_{2,0}^{\prime}$ is an invariant operator. By virtue of Eq. (74), however, $V_{2,0}^{\prime}$ is expressed in terms of commutators that have zero spectrum. Hence, $V_{2,0}^{\prime}$ has zero spectrum and must vanish, $V_{2,0}^{\prime}=0$. Collecting all terms, we obtain the final result

$$
\begin{aligned}
V_{2}= & \mathbb{T}_{A-V}^{(3), \text { inv }}+\beta_{0} \mathbb{T}_{A-V}^{(2)}+\frac{1}{2} \mathbb{T}_{V}^{(1)} \mathbb{\boxplus}_{A-V}^{(2)}+\frac{1}{2} \mathbb{T}_{A-V}^{(2)} \mathbb{\boxplus}_{V}^{(1)} \\
& +\left[\mathbb{G}_{A-V}^{(2)}, \mathbb{X}_{V}^{(1)}\right] .
\end{aligned}
$$

Note that Eq. (74) was not used to derive the expressions in (76)-(78) and provides a highly nontrivial consistency check,

$$
\begin{aligned}
0= & \frac{1}{4}\left[\mathbb{W}_{V}^{(1)}, V_{2,1}^{\prime}\right]+\left[\mathbb{W}_{V}^{(1)}, \Delta \mathbb{X}_{A-V}^{(2)}\right]-\frac{1}{4 \beta_{0}}\left[\mathbb{T}_{A-V}^{(2)}, \mathbb{T}_{V}^{(1)}\right] \mathbb{H}_{V}^{(1)} \\
& -\frac{1}{2 \beta_{0}}\left[\mathbb{G}_{A-V}^{(2)},\left[\mathbb{G}_{V}^{(1)}, \mathbb{X}_{V}^{(1)}\right]\right] .
\end{aligned}
$$

Using $V_{2,1}^{\prime}$ from Eq. (77), the expression on the rhs of this relation can be brought to the form $\left[\mathbb{q}_{V}^{(1)}, \mathbb{F}\right]$ where
We have verified that this equation holds true. Alternatively, Eq. (82) can be used to obtain the conformal anomaly $\hat{\Delta}_{A-V}^{(2)}$ avoiding the diagrammatic calculation described in Sec. IV.

The result for the matching kernel in Eq. (79) can be written as an integral operator in momentum fraction representation. This is, however, not necessary as the matching can be implemented more efficiently starting from position-space kernels as explained in Ref. [15]. For the applications to LCDAs, the expansion in local operators is more useful. We derive the corresponding expressions in the Appendix.

\section{SUMMARY}

We have calculated the three-loop evolution kernel for flavor-nonsinglet axial-vector operators in general offforward kinematics in Larin's scheme. In QCD applications, axial-vector operators are usually defined in such a way that their scale dependence coincides with that for the vector operators, which can be achieved starting from Larin's scheme and applying a finite renormalization (matching) to arrive at the conventional choice for the $\overline{\mathrm{MS}}$ scheme. Of course, the coefficient functions in the OPE have to be modified accordingly. We have derived the explicit expression for the matching kernel for the light-ray operators and also for local operators.

Our method of calculation is based on using the restrictions on off-forward operator mixing that are due to conformal symmetry of the QCD Lagrangian and can most naturally be taken into account going over to the Wilson-Fisher critical point at noninteger $d=4-2 \varepsilon$ space-time dimensions. In this work, the technique has been generalized to axial-vector operators and we have derived the two-loop expression for the special conformal anomaly for axial-vector operators.

The results are relevant for QCD studies of hard exclusive reactions involving momentum transfer between the initial and the final states, e.g., DVCS and $\gamma^{*} \gamma \rightarrow \pi$ form 
factor. Concrete applications are beyond the scope of this study.

\section{ACKNOWLEDGMENTS}

This project was supported by Deutsche Forschungsgemeinschaft (DFG) through the Research Unit FOR 2926, "Next Generation pQCD for Hadron Structure: Preparing for the EIC," Project No. 40824754. The work of A. M. was supported in addition by the DFG Grants No. MO 1801/4-1 and No. KN 365/13-1 and RSF Project No. 19-11-0013.

\section{APPENDIX: MATCHING KERNEL FOR LOCAL OPERATORS}

Light-ray operators are nothing but the generating functions for the renormalized local operators. The results in the local form are required for several applications, e.g., the calculation of moments of the LCDAs and GPDs using lattice QCD techniques. Our notations in this Appendix closely follow Sec. VI in Ref. [14].

Instead of using mixing matrices for the operators with a given number of left and right derivatives, it proves to be more convenient to go over to the Gegenbauer polynomial basis,

$$
\mathcal{O}_{n k}^{A}=\left.\left(\partial_{z_{1}}+\partial_{z_{2}}\right)^{k} C_{n}^{3 / 2}\left(\frac{\partial_{z_{1}}-\partial_{z_{2}}}{\partial_{z_{1}}+\partial_{z_{2}}}\right) \mathcal{O}_{A}\left(z_{1}, z_{2}\right)\right|_{z_{i}=0} .
$$

Here $k \geq n$ is the total number of derivatives. The rationale for using Gegenbauer polynomials is that any invariant kernel is diagonal in this basis. Note that the Lorentz spin of the operator with the lowest dimension for given $n$ is $N=n+1$.

The RG equation for the operators $\mathcal{O}_{n k}^{A}$ has the form

$$
\left(\mu \frac{\partial}{\partial \mu}+\beta(a) \frac{\partial}{\partial a}\right) \mathcal{O}_{n k}^{A}=-\sum_{n^{\prime}=0}^{n} \gamma_{n n^{\prime}}^{A} \mathcal{O}_{n^{\prime} k}^{A}
$$

The mixing matrix $\gamma_{n n^{\prime}}^{A}$ is triangular and its diagonal elements are equal to the anomalous dimensions

$$
\gamma_{n n^{\prime}}^{A}=0 \quad \text { if } n^{\prime}>n, \quad \gamma_{n n}^{A}=\gamma_{A}(n+1) .
$$

The light-ray operator can be expanded over local operators as follows:

$$
\mathcal{O}_{A}\left(z_{1}, z_{2}\right)=\sum_{n=0}^{\infty} \sum_{k=n}^{\infty} \Phi_{n k}\left(z_{1}, z_{2}\right) \mathcal{O}_{n k}^{A}
$$

The coefficients $\Phi_{n k}\left(z_{1}, z_{2}\right)$ in this expansion are homogeneous polynomials in $z_{1}, z_{2}$ of degree $k$,

$$
\Phi_{n k}\left(z_{1}, z_{2}\right)=\omega_{n k}\left(S_{+}\right)^{k-n} z_{12}^{n} .
$$

The normalization factor reads [14]

$$
\omega_{n k}=2 \frac{2 n+3}{(k-n) !} \frac{\Gamma(n+2)}{\Gamma(n+k+4)} .
$$

The action of any integral operator $\mathbf{A}$ on the light-ray operator $\mathcal{O}_{A}$ can be translated into the matrix form

$$
\begin{aligned}
{\left[\mathbf{A} \mathcal{O}_{A}\right]\left(z_{1}, z_{2}\right) } & =\sum_{n k}\left[\mathbf{A} \Phi_{n k}\right]\left(z_{1}, z_{2}\right) \mathcal{O}_{n k}^{A} \\
& =\sum_{n k} \Phi_{n k}\left(z_{1}, z_{2}\right) \sum_{n^{\prime} k^{\prime}} A_{n n^{\prime}}^{k k^{\prime}} \mathcal{O}_{n^{\prime} k^{\prime}}
\end{aligned}
$$

where the matrix $A_{n n^{\prime}}^{k k^{\prime}}$ is defined as

$$
\left[\mathbf{A} \Phi_{n k}\right]\left(z_{1}, z_{2}\right)=\sum_{n^{\prime} k^{\prime}} A_{n^{\prime} n}^{k^{\prime} k} \Phi_{n^{\prime} k^{\prime}}\left(z_{1}, z_{2}\right)
$$

See Ref. [14] [Sect. 6] for more details. Here we only note that if the operator A commutes with the canonical generator of scale transformations $S_{0}$, then its matrix elements are nonzero only if $k=k^{\prime}, A_{n n^{\prime}}^{k k^{\prime}} \equiv \delta_{k k^{\prime}} A_{n n^{\prime}}(k)$. If, in addition, A commutes with $S_{-}$, then the matrix elements $A_{n n^{\prime}}$ do not depend on $k, A_{n n^{\prime}}(k)=A_{n n^{\prime}}$.

Our goal in this Appendix is to work out this representation for the matching kernel (64), $V(a) \mapsto[V(a)]_{n n^{\prime}}^{k k^{\prime}}$. Since the operator $V(a)$ satisfies the both requirements, the $k$-indices are redundant,

$$
[V(a)]_{n n^{\prime}}^{k k^{\prime}}=\delta_{k k^{\prime}} V_{n n^{\prime}}(a) .
$$

Following [8], we split $V_{m n}$ in the diagonal $m=n$ and nondiagonal $n>m$ parts,

$$
V_{m n}(a)=\delta_{m n} V_{n}^{\mathrm{D}}(a)+V_{m n}^{\mathrm{ND}}(a) .
$$

The diagonal part is given in terms of the difference in the anomalous dimensions,

$$
V_{n}^{\mathrm{D}}(a)=a \gamma_{A-V}^{(2)}(n+1)+a^{2} \gamma_{A-V}^{(3)}(n+1)+\cdots .
$$

The leading contribution is

$$
\gamma_{A-V}^{(2)}(n+1)=\frac{16 C_{F} \beta_{0}}{(n+1)(n+2)} .
$$

The three-loop result $\gamma_{A-V}^{(3)}(n+1)=\gamma_{A-V}^{(3)}(N)$ is available from Ref. [22] and we have presented it in the form

$\gamma_{A-V}^{(3)}(n+1)=\gamma_{A-V}^{(3), \text { inv }}(n+1)+\gamma_{A-V}^{(3), \text { ninv }}(n+1)$. 
See Eqs. (53) and (55) for the explicit expressions. The offdiagonal part of the matching matrix

$$
V_{m n}^{\mathrm{ND}}(a)=a^{2} V_{m n}^{(2), \mathrm{ND}}+O\left(a^{3}\right)
$$

is a new result. We obtain

$$
\begin{aligned}
V_{m n}^{(2), \mathrm{ND}}= & -\frac{1}{a(m, n)}\left\{\left(\gamma_{A-V}^{(2)}(m+1)-\gamma_{A-V}^{(2)}(n+1)\right)\right. \\
& \times\left(\left(\beta_{0}+\frac{1}{2} \gamma_{V}^{(1)}(n+1)\right) \mathbf{b}_{m n}+\mathbf{w}_{m n}^{(1)}\right) \\
& \left.+\frac{1}{2}\left(\gamma_{V}^{(1)}(m+1)-\gamma_{V}^{(1)}(n+1)\right) \gamma_{A-V}^{(2)}(n+1) \mathbf{b}_{m n}\right\} .
\end{aligned}
$$

Here

$$
a(m, n)=(m-n)(m+n+3),
$$

and the matrices $\mathbf{b}_{m n}, \mathbf{w}_{m n}^{(1)}$ have the form [14]

$$
\begin{gathered}
\mathbf{b}_{m n}=-2(2 n+3) \vartheta_{m n}, \\
\mathbf{w}_{m n}^{(1)}=4 C_{F}(2 n+3) a(m, n) \\
\times\left(\frac{A_{m n}-S_{1}(m+1)}{(n+1)(n+2)}+\frac{2 A_{m n}}{a(m, n)}\right) \vartheta_{m n},
\end{gathered}
$$

where

$$
\vartheta_{m n}= \begin{cases}1 & \text { if } m-n>0 \text { and even } \\ 0 & \text { else }\end{cases}
$$

and

$$
\begin{aligned}
A_{m n}= & S_{1}\left(\frac{m+n+2}{2}\right)-S_{1}\left(\frac{m-n-2}{2}\right) \\
& +2 S_{1}(m-n-1)-S_{1}(m+1) .
\end{aligned}
$$

The first few nondiagonal elements $(0 \leq m, n \leq 7)$ for $N_{c}=3$ and $n_{f}=4$ are equal to

$$
V^{2, \mathrm{ND}}=\left(\begin{array}{ccccccc}
0 & 0 & 0 & 0 & 0 & 0 & 0 \\
0 & 0 & 0 & 0 & 0 & 0 & 0 \\
\frac{2000}{9} & 0 & 0 & 0 & 0 & 0 & 0 \\
0 & \frac{2800}{729} & 0 & 0 & 0 & 0 & 0 \\
\frac{4640}{27} & 0 & -\frac{1120}{81} & 0 & 0 & 0 & 0 \\
0 & \frac{52000}{1701} & 0 & -\frac{12512}{945} & 0 & 0 & 0 \\
\frac{29800}{243} & 0 & \frac{1580}{243} & 0 & -\frac{4312}{405} & 0 & 0
\end{array}\right) .
$$

[1] J. Dudek et al., Physics opportunities with the $12 \mathrm{GeV}$ upgrade at Jefferson Lab, Eur. Phys. J. A 48, 187 (2012).

[2] W. Altmannshofer et al. (Belle-II Collaboration), The Belle II physics book, Prog. Theor. Exp. Phys. 2019, $123 \mathrm{C} 01$ (2019); Erratum, Prog. Theor. Exp. Phys. 2020, 029201 (2020).

[3] A. Accardi et al., Electron ion collider: The next QCD frontier: Understanding the glue that binds us all, Eur. Phys. J. A 52, 268 (2016).

[4] S. Moch, J. Vermaseren, and A. Vogt, The three loop splitting functions in QCD: The nonsinglet case, Nucl. Phys. B688, 101 (2004).

[5] A. Vogt, S. Moch, and J. Vermaseren, The three-loop splitting functions in QCD: The singlet case, Nucl. Phys. B691, 129 (2004).

[6] D. Müller, Restricted conformal invariance in QCD and its predictive power for virtual two photon processes, Phys. Rev. D 58, 054005 (1998).

[7] A. V. Belitsky and D. Müller, Next-to-leading order evolution of twist-2 conformal operators: The Abelian case, Nucl. Phys. B527, 207 (1998).

[8] A. V. Belitsky and D. Müller, Broken conformal invariance and spectrum of anomalous dimensions in QCD, Nucl. Phys. B537, 397 (1999).
[9] D. Müller, Constraints for anomalous dimensions of local light cone operators in $\phi^{3}$ in six-dimensions theory, Z. Phys. C 49, 293 (1991).

[10] V. Braun and A. Manashov, Two-loop evolution equations for light-ray operators, Phys. Lett. B 734, 137 (2014).

[11] V. Braun, A. Manashov, S. Moch, and M. Strohmaier, Twoloop evolution equations for flavor-singlet light-ray operators, J. High Energy Phys. 02 (2019) 191.

[12] V. Braun and A. Manashov, Evolution equations beyond one loop from conformal symmetry, Eur. Phys. J. C 73, 2544 (2013).

[13] V. Braun, A. Manashov, S. Moch, and M. Strohmaier, Twoloop conformal generators for leading-twist operators in QCD, J. High Energy Phys. 03 (2016) 142.

[14] V. Braun, A. Manashov, S. Moch, and M. Strohmaier, Three-loop evolution equation for flavor-nonsinglet operators in off-forward kinematics, J. High Energy Phys. 06 (2017) 037.

[15] V. Braun, A. Manashov, S. Moch, and J. Schönleber, Twoloop coefficient function for DVCS: Vector contributions, J. High Energy Phys. 09 (2020) 117.

[16] S. Larin, The renormalization of the axial anomaly in dimensional regularization, Phys. Lett. B 303, 113 (1993). 
[17] D. Akyeampong and R. Delbourgo, Dimensional regularization, abnormal amplitudes and anomalies, Nuovo Cimento A 17, 578 (1973).

[18] S. Larin and J. Vermaseren, The $\alpha_{s}^{3}$ corrections to the Bjorken sum rule for polarized electroproduction and to the GrossLlewellyn Smith sum rule, Phys. Lett. B 259, 345 (1991).

[19] E. Zijlstra and W. van Neerven, Order $\alpha_{s}^{2}$ correction to the structure function $F_{3}\left(x, Q^{2}\right)$ in deep inelastic neutrinohadron scattering, Phys. Lett. B 297, 377 (1992).

[20] Y. Matiounine, J. Smith, and W. van Neerven, Two loop operator matrix elements calculated up to finite terms for polarized deep inelastic lepton-hadron scattering, Phys. Rev. D 58, 076002 (1998).

[21] S. Moch, J. Vermaseren, and A. Vogt, On $\gamma_{5}$ in higher-order QCD calculations and the NNLO evolution of the polarized valence distribution, Phys. Lett. B 748, 432 (2015).

[22] S. Moch, J. Vermaseren, and A. Vogt, The three-loop splitting functions in QCD: The helicity-dependent case, Nucl. Phys. B889, 351 (2014).

[23] A. Behring, J. Blümlein, A. De Freitas, A. Goedicke, S. Klein, A. von Manteuffel, C. Schneider, and K. Schönwald, The polarized three-loop anomalous dimensions from on-shell massive operator matrix elements, Nucl. Phys. B948, 114753 (2019).

[24] I. Balitsky and V. M. Braun, Evolution equations for QCD string operators, Nucl. Phys. B311, 541 (1989).

[25] Note that equations of the type $\left[S_{+}, \mathcal{O}\right](z)=\mathcal{G}(z)$ are nothing but first-order inhomogeneous differential equations, whose solutions are defined up to an arbitrary invariant operator, $\left[S_{+}, \mathcal{O}^{\text {inv }}\right]=0$. Clearly, this ambiguity only results in a redefinition of $\mathrm{H}_{A-V}^{(3) \text {,inv }}$ in Eq. (26).

[26] J. Vermaseren, Harmonic sums, Mellin transforms and integrals, Int. J. Mod. Phys. A 14, 2037 (1999).

[27] Y. Dokshitzer, G. Marchesini, and G. Salam, Revisiting parton evolution and the large-x limit, Phys. Lett. B 634, 504 (2006).

[28] B. Basso and G. Korchemsky, Anomalous dimensions of high-spin operators beyond the leading order, Nucl. Phys. B775, 1 (2007).

[29] L. F. Alday, A. Bissi, and T. Lukowski, Large spin systematics in CFT, J. High Energy Phys. 11 (2015) 101.

[30] L. F. Alday and A. Zhiboedov, An algebraic approach to the analytic bootstrap, J. High Energy Phys. 04 (2017) 157. 